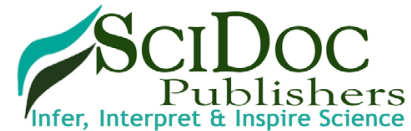

International Journal of HIV/AIDS and Research (IJHR)

ISSN 2379-1586

\title{
The Relationships between Cholesterol, Tobacco and Alcohol Use : A New Opportunity for Intervention among People Living with HIV
}

Research Article

Míguez MJ", Rosenberg R, Perez C, Espinoza L, Yoo C, Vargas M

Florida International University, USA.

Abstract

Objective: Given increased evidence of cholesterol's role in the structure and functioning of the central nervous system, this study examines how lipid disturbances might deregulate drinking behaviors.

Methods: Using a clinical cohort, the relationship between hypocholesterolemia and alcohol use was prospectively assessed over a period of 6 months while controlling for biological, familial, and intrapersonal factors. People Living with HIV (PLWH) were stratified according to baseline cholesterol levels: hypocholesterolemic (HypoCHL $<150 \mathrm{mg} / \mathrm{dl}$ ) or nonHypoCHL $(>150 \mathrm{mg} / \mathrm{dl})$. We used a Bayesian network model to analyze the probability that HypoCHL predicts alcohol trajectories.

Results: At baseline, those participants with HypoCHL consumed more alcohol (29.9 \pm 4 vs. $15.1 \pm 2$ drinks $p=0.001)$ and on more days/week ( $4 \pm 2.7$ vs. $2.8 \pm 2.5, p=0.002)$ than normal CHL participants. At the follow-up evaluation, alcohol consumption increased or remained constant in $60 \%$ of the sample and was associated with four risk factors: HypoCHL $(\mathrm{OR}=1.5, \mathrm{p}=0.04)$, cigarette smoking $(\mathrm{OR}=2.9, p=0.008)$, drinking liquor $(\mathrm{OR}=1.9, \mathrm{p}=0.05)$, and having a close relative with hazardous alcohol use $(\mathrm{OR}=3, \mathrm{p}=0.0001)$. The Bayesian model further predicted that if a subject has a baseline cholesterol level below $150 \mathrm{mg}$, that there would be an $87 \%$ probability of maintaining or increasing alcohol intake, and only a $14 \%$ chance of reducing intake during the study. Noteworthy, if cholesterol was modeled to be normal, the probability of reducing alcohol intake increased to $41 \%$.

Conclusion: This study provides evidence that HypoCHL is a significant predictor of increasing or continuing hazardous consumption of alcohol in PLWH.

Keywords: Alcohol; Cholesterol (HypoCHL); HIV; Neurocognitive; Trajectories; Hazardous Alcohol Use (HAU); People living with HIV/AIDS (PLWH).

\section{Introduction}

Hazardous Alcohol Use (HAU) is recognized as a worldwide epidemic. In 2011, the World Health Organization (WHO) reported that the harmful use of alcohol resulted in 2.5 million deaths annually, and ranked as the third leading risk factor contributing to the global burden of disease, followed by unsafe sexual behaviors and being underweight in childhood [1]. Approximately 17.6 million (8.5\%) adults meet the diagnostic criteria for alcohol use disorders in the United States. Although alcohol use is common throughout the U.S. population, data has shown that the rates are even higher among People Living with HIV (PLWH), significantly complicating prognosis and diminishing an individual's quality of life $[1,2]$. Additional evidence shows that
HAU interferes with adherence and antiretroviral drug response, accelerating disease progression and promoting liver failure [2-9]. Due to the fact that HAU is a major avoidable risk factor in global disease and disability, particularly among PLWH, this topic poses high relevance to future HIV care and treatment. Clarification of this issue is also necessary to achieve maximum benefits for the success and sustainability of our efforts to control transmission and community viral load [10].

Cognitive, biochemical, and psychopathological characteristics are regularly documented in relation to alcohol consumption [11-15]. Notably, these same bio-behavioral features have been reported in subjects with hypercholesterolemia [16-22]. Several lines of scientific evidence have documented a close relationship

\footnotetext{
*Corresponding Author:

Maria Jose Miguez-Burbano, MD, PhD

Professor, Florida International University/School of Integrated Sciences and Humanity, AHC4 - 352, 11200 S.W. 8th St., Miami, FL 33199, USA.

E-mail: mjmiguez@fiu.edu
}

Received: June 28, 2016

Accepted: July 26, 2016

Published: July 28, 2016

Citation: Míguez MJ, Rosenberg R, Perez C, Espinoza L, Yoo C, Vargas M (2016) The Relationships between Cholesterol, Tobacco and Alcohol Use : A New Opportunity for Intervention among People Living with HIV. Int J AIDS Res. 3(5), 78-86. doi: http://dx.doi.org/10.19070/2379-1586-1600017

Copyright: Míguez $\mathbf{M J}^{\circ}$ 2016. This is an open-access article distributed under the terms of the Creative Commons Attribution License, which permits unrestricted use, distribution and reproduction in any medium, provided the original author and source are credited. 
between hypocholesterolemia and mood disorders [15-18]. Numerous studies among the general population have also recognized the deleterious effects of HypoCHL on cognitive performance, findings that we have replicated in PLWH [18-24]. These findings are not unexpected, as studies have indicated that a decrease in brain synaptosomal membrane cholesterol results in pronounced alterations in brain serotonin and dopamine [2527]. Since serotonergic dysfunction has been implicated in the pathophysiology underlying an individual's drinking behavior, serotonin down-regulation would be expected to produce profound influences on drinking behaviors, poorer outcomes regarding alcohol treatment prognosis, and treatment dropouts [9, 25-28]. Noteworthy, in animals, neurological adaptation (tolerance) to chronic alcohol administration has been associated with increased cholesterol in cell membranes [27]. To date, however, such findings have not yet been replicated in humans.

Given CHL's effects on neuropsychological well-being and the neuromodulators described above, the pressing question is whether a relationship between cholesterol level and alcohol consumption exists, and if so, if that can be used to develop more effective evidence-based initiatives. In seeking to answer these paradigms and to test our proposed model (depicted in Figure 1), a longitudinal study with PLWH was performed.

\section{Methods}

\section{Subjects, Setting and Procedures}

MARCH (Miami Alcohol Research Cohort of HIV+) was a longitudinal, observational study to evaluate the impact of hazardous versus non-hazardous alcohol use and alcohol use trajectories on the health status of individuals initiating highly active antiretroviral therapy (HAART). The MARCH study consists of 150 PLWH who were consecutively enrolled if they had been at least 18 years old and receiving regular care at Miami's primary open-access public health system (Jackson Memorial Medical Center) for treatment of their HIV. Our recruitment of PLWH in an open-access public health system with standard treatment protocols was primarily chosen to minimize social, medical, and treatment inequalities that can sometimes confound outcomes. Subjects were enrolled regardless of their CDC defined HIV status. Nonetheless, the distribution of participants across CDC categories was well-balanced, with $48 \%$ of the group fulfilling the criteria for AIDS.

Non-ambulatory patients were excluded, as well as those with major comorbidities, including but not limited to, CNS opportunistic infections, head injuries with or without loss of consciousness, tumors, major psychiatric diseases, developmental disorders, severe malnutrition or confirmed cardiovascular or immune based disease (i.e., malignancies, autoimmune diseases, arthritis). Given that our main goal was to establish the health effects of alcohol, dependent drug users and subjects reporting injection drug use were excluded to reduce their confounding effects. We also excluded any participants who had cirrhosis, active viral hepatitis, or liver enzymes two standard deviations above the normal values.

Those participants who expressed a willingness to participate and to provide written informed consent, as well as a medical release, were consecutively enrolled and followed over a period of 6 months. The Institutional Review Board at the University of Miami approved the study. Upon enrollment, participants completed standardized research questionnaires, a brief physical exam, and laboratory tests. Of the total 150, 130 participants completed the two required visits and fulfilled the data portion of the study. Five other subjects were excluded from the analyses because they were taking lipid lowering medications or were on a diet regimen.

\section{Outcome Variable: Alcohol Use}

At each visit, participants reported alcohol intakes in the past six months using two standardized and validated brief screening questionnaires: The Alcohol Use Disorders Identification Test (AUDIT) and the Alcohol Dependence Scale (ADS). The latter assesses alcohol withdrawal symptoms, impaired control over drinking, awareness of a compulsion to drink, increased tolerance to alcohol, and salience of drink-seeking behavior [29-32].

Alcohol consumption scores for each beverage were computed by: 1) transforming the responses to standardized alcohol units, and 2) averaging cross products of the quantity and frequency of beer, wine, and hard liquor.

Consumption phenotypes related to heavy drinking can be broken down into two categories: (1) consumption of 'too much too fast'

Figure 1. Hypocholesterolemia Proposed Model.

\section{LOW CHOLESTEROL}

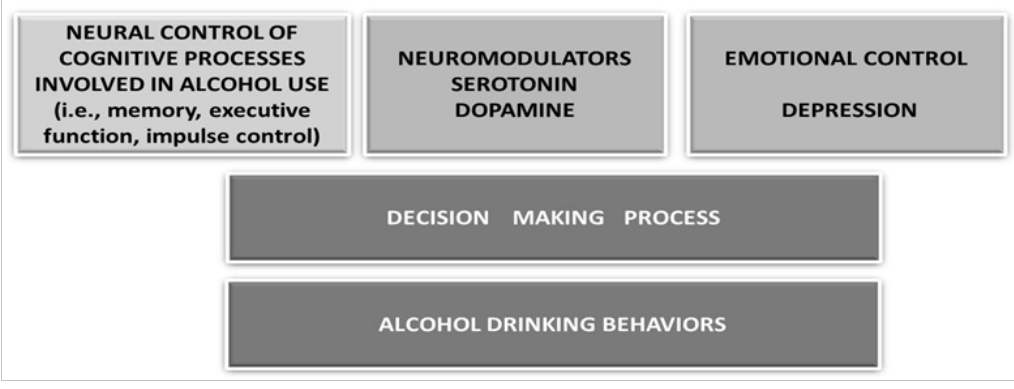

Legend:Our model proposes a direct effect of low cholesterol over neuromodulators, neurological, and emotional status. This can lead to high-risk alcohol drinking behaviors directly or by impacting decision-making. 
and (2) consumption of 'too much too often' [29-32]. The first phenotype known as "heavy episodic drinking" was established a decade ago and was defined as five or more drinks in a day for males and four or more drinks in a day for females (i.e. the '5/4 definition'). The second phenotype refers to heavily drinking a higher quantity of alcohol than is recommended on a regular basis (i.e. 'too much too often'). Following the National Institute of Alcohol Abuse and Alcoholism guidelines criteria, men who reported $>14$, and women $>7$ drinks/week were classified into the hazardous drinkers group, while those who reported fewer drinks were included in the non-hazardous drinkers group [2932]. Avariable was created to analyze drinking trajectories. The variable was the result of subtracting baseline total number of drinks consumed per week from the 6-month values. Based on individual trajectories, each participant was assigned to one of three mutually exclusive groups: 1) stable consumption; 2) increased consumption; and finally, 3) decreased consumption.

\section{Cholesterol}

Although for years it has been argued that the cholesterol content in the brain is fully independent of the systemic pool, there is increasing evidence to show the exchange of cholesterol between the brain and the blood pool [33]. Furthermore, data derived from animal models clearly demonstrates that a low, but continuous flux of sterol synthesis and export is required for normal CNS function. Given these findings and the limitations on obtaining direct CNS measures, we acquired fasting blood samples at baseline and at the 6 months follow-up. Total cholesterol (TC) levels were measured by routine enzymatic methods (KonePro, Konelab). In addition to treating mean TC values as a continuous variable, subjects were categorized as having or not having hypocholesterolemia. Hypocholesterolemia (levels $<150 \mathrm{mg} / \mathrm{dL}$ ) was defined according to the US National Cholesterol Education Program guidelines [34].

\section{Plausible Confounding Factors}

Information derived from income, education, age, race, and gender was considered in our study. These variables have been associated with drinking patterns previously. A family history of alcohol abuse was obtained as part of the initial assessments and was classified as yes/no. We also assessed family, partner, and/or friend support regarding HIV disease, treatment adherence, and alcohol related problems (ACTG, ADS). Due to the relationship between alcohol use and other drug risk behaviors, tobacco and drug use habits were assessed using standardized questionnaires.

Given the potentially confounding effects of health status on our outcome assessment, to measure and control for this effect in our analyses, we have assessed several general health status variables, such as anthropometrics, albumin and body mass index along with specific markers of HIV disease status. Weight was measured to the nearest $0.1 \mathrm{~kg}$ on a balance beam scale, with participants dressed in indoor clothing and without shoes. Height was measured to the nearest $0.1 \mathrm{~cm}$, using a wall-mounted stadiometer. Body mass index (BMI) was calculated as weight in kilograms divided by height in meters squared. In addition, a detailed current and past medical history was obtained on each participant. Depression was assessed with the Beck Depression Inventory (BDI) form, a widely used measure with good internal consistency (coefficient alpha $=0.8$ ), and adequate construct validity [31]. Each of the 21 items is rated on a 4-point Likert scale, ranging from 0 (neutral) to 3 (maximum severity), and summed to obtain a total score (0 to 63). Participants with BDI scores over 19 were classified as either having moderate to severe depression $[35,36]$.

Using flow cytometry,as per the National Institute of Allergy and Infectious Diseases laboratory protocols, CD 4 cell counts were assessed in freshly obtained whole blood. To assess the degree of HIV activity, viral load was assessed by an Amplicor HIV-1 monitor kit, using reverse transcriptase polymerase chain reaction (COBAS Amplicor Analyzer [Roche Molecular Diagnostics, Pleasanton, CA]).

\section{Statistical Analyses}

Data sets were analyzed using SAS 8.1 (SAS, Institute Inc., Cary, NC, USA) and SPSS 18 (SPSS, Inc., Chicago, IL, USA). Associations between the main variables of interest were examined with Pearson's correlation coefficient analyses. Subgroups were compared using analysis of variance.

Several approaches were used to investigate drinking trajectories. The first analyses involved a paired Student's t test to compare changes from T1 to T2. The non-parametric Wilcoxon test was also used for these comparisons, as normality of the distributions could not be presumed in all of the cases. To prepare data for the second trajectory analysis, we subtracted baseline number of total drinks per week from those in the last visit, identifying three distinctive groups:1) those who increased alcohol consumption, 2) those who decreased consumption, and 3) those whose consumption levels remained stabilized.

Multivariate regression analysis was used to describe the associations between cholesterol and alcohol drinking trajectories, while controlling for all variables that were statistically significant in the univariate analyses. Multiple logistic regression analyses were used to evaluate the effects of cholesterol, and other potential risk factors on alcohol drinking. The initial model was constructed with age, socioeconomic status, body mass index, dementia and depression, as predictive variables. These factors were selected because they were significant $(p=0.05)$ in the univariate analysis, or they were recognized risk factors (i.e. socio-demographics, global markers of health status, mood and cognitive disorders).

In all models, we adjusted for any baseline alcohol intake differences so as not to have this variable as a possible explanation of differences between trajectory groups in drinking. Nonsignificant variables were removed, beginning with the least significant variables, until the final full model was determined. Regression output was reported as adjusted OR or RR when analyzing longitudinal data accompanied by $95 \%$ CI.

Additionally, we applied Bayesian Networks learning method to further understand interactions among the variables of interest.A Bayesian network is a probabilistic model that consists of two parts: a dependency structure and a probability model [37, 38]. The probability model specifies how variables depend on others. Usually, a variable only depends on a few other variables, called the parents. We evaluated (scored) a Bayesian Network using the following assumptions: (1) discrete variables, (2) Dirichlet prior parameter distributions, (3) multinomial likelihood functions; (4) parameter independence; (5) parameter modularity, and (6) 
no missing data [37, 38]. A stage-wise approach was used to eliminate variables that ceased to be significant in the models. The probability model specifies the probability that a variable takes a certain value, given the value of its parents. Each statistical model assessed interactions between the significant variables and time.

\section{Results}

\section{Characteristics of the Study Population}

MARCH was designed as a longitudinal, observational study to evaluate the impact of hazardous versus non-hazardous alcohol use on the health status of HIV-infected individuals receiving HAART therapy. With an overall participation rate of $98 \%$ and a retention rate of $85 \%$, the sample included 125 participants who did not receive cholesterol-lowering medication, and who had their blood drawn at both the baseline and six-month follow-up periods. The mean TC level of the sample was $173 \pm 43 \mathrm{mg} / \mathrm{dl}$ (52-324mg/dl), with HypoCHL present in $40 \%$ of participants at baseline, and $33 \%$ at week 24 . Only $5 \%$ had more than $250 \mathrm{mg} /$ $\mathrm{dl}$ of TC. Cholesterol levels were unrelated to triglyceride levels and/or to malnutrition.

Table 1 displays the demographic and clinical characteristics of the sample grouped by cholesterol. Groups were comparable on socio-demographic variables. Patients with HypoCHL were less likely to be White (95\% CI: $0.0-0.48, p=0.001)$. In addition, we did not observe any differences in vitamin B-12 levels between hazardous and non-hazardous alcohol users, nor between subjects with low versus normal cholesterol levels (HypoCHL: $615 \pm 394$ vs. $674.2 \pm 422$ ). These findings are highly relevant because it indicates that the groups were similar, thus reducing the possibility that nutritional status/dietary habits may explain the observed differences on either cholesterol or neuropsychological functions. These results are also highly pertinent in light of studies indicating that poor learning and memory performance follow a detoxification predicted relapse [39]. Although evidence exists that poor health (e.g., malnutrition) is associated with both HAU and hypocholesterolemia, our analyses failed to uncover a significant association between hypocholesteromia and vitamin B-12 levels or malnutrition (see Table 1), suggesting that hypocholesterolemia was not the result of frailty. Furthermore, we did not find any differences in dietary intakes.

However, participants with HypoCHL had a 58\% excess risk of having depressive symptoms (95\% CI 1-3, $\mathrm{p}=0.05)$. Univariate analyses indicated that those with baseline depression were seven times more likely to increase the number of drinks/day, compared to those without any depressive symptoms (95\% CI 1-10; $p=0.05$ ).

\section{Cholesterol (CHL) Status and Alcohol Drinking Trajectories}

Drinking was common in the sample, varying from 0-100 drinks per week $(31 \pm 24$ drinks/week). Almost half of the study population was comprised of hazardous alcohol users (48\%), and despite receiving HAART, the participants reported an average alcohol consumption of $25 \pm 2.5$ drinks/week. Similar to prior studies, analyses indicated that smokers consume significantly higher amounts of alcohol on a weekly basis $(23.9 \pm 2.4$ vs. $15.5 \pm 3.1, p=0.04)$ and in a single occasion $(17.2 \pm 1.4$ vs. $9.8 \pm$ $1.4, p=0.008)$ but did not significantly differ in either CAGE or AUDIT scores. Univariate analyses indicated that smokers were twice more likely to be hazardous alcohol users than non-smokers $(\mathrm{OR}=2$ 95\% CI 1.1-4, $p=0.01)$.

At baseline, participants with HypoCHL consumed significantly

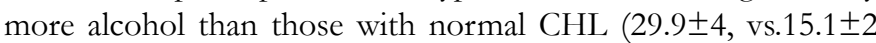
$3 \mathrm{p}=0.001)$. Compared to normal CHL participants, those with HypoCHL consumed alcohol on more days during the week (4 \pm 2.7 vs. $2.8 \pm 2.5$ days/week, $\mathrm{p}=0.002$ ). Our analyses also revealed a significant difference in maximum number of drinks consumed on a single occasion between participants with HypoCHL

Table 1. Sociodemographic Information of HIV-Infected Patients with and without Hypocholesterolemia (Baseline).

\begin{tabular}{|c|c|c|}
\hline Variables & Hypocholesterolemia & $\begin{array}{c}\text { Normal Total Cho- } \\
\text { lesterol Levels }\end{array}$ \\
\hline Age & $39.8 \pm 7.9$ & $41.1 \pm 7.2$ \\
\hline Men & $72 \%$ & $68 \%$ \\
\hline Women & $28 \%$ & $32 \%$ \\
\hline Black & $65 \%$ & $5 \%$ \\
\hline Hispanic & $33 \%$ & $29 \%$ \\
\hline White & $0 \%$ & $16 \%$ \\
\hline Less than $\$ 10,000$ & $89 \%$ & $95 \%$ \\
\hline \$11,000-\$20,000 & $9 \%$ & $2 \%$ \\
\hline More than \$20,000 & $2 \%$ & $3 \%$ \\
\hline Tobacco & $81 \%$ & $80 \%$ \\
\hline No & $19 \%$ & $20 \%$ \\
\hline Albumin & $4.0 \pm 0.4$ & $4.1 \pm 0.5$ \\
\hline Body Mass Index & $26.2 \pm 6.2$ & $27.4 \pm 6.7$ \\
\hline Vitamin B 12 & $612.8 \pm 462$ & $682.5 \pm 462$ \\
\hline
\end{tabular}

Legend:Values are means \pm SD or percentages.

No significant differences in sociodemographic characteristics were found between groups except for race. 
and those with normal CHL $(18 \pm 2$ vs. $12 \pm 1, p=0.015)$. Furthermore, participants with HypoCHL exhibited significantly higher AUDIT scores than their normal CHL counterparts $(9.2 \pm$ 0.7 vs. $7.3 \pm 0.7, p=0.05)$. Notably, no differences in smoking rates were observed between cholesterol groups. Because the effects of advancing HIV disease might confound our results, we repeat the analyses in individuals with and without AIDS and found no differences in the results.

Surprisingly, at the follow-up evaluation after 6 months postHAART, most drinkers had significantly reduced the total number of alcohol drinks/week (from 32.9 drinks/week to 15.5, $p=0.005$ ) and maximum number of drinks consumed on any one occasion (16.8 max. to 12.5). Therefore, we performed a trajectory analysis and were able to identify three distinctive trajectories regardless of initial drinking levels. Overall, a significant subgroup (40\%) either decreased or quit drinking, 15\% maintained a constant level, and the remaining sample (45\%) increased drinking throughout the duration of the study.

As illustrated in Figure 2, the drinking trajectories clearly differed between those having hypocholesterolemia versus normal values at baseline. Compared to those with normal baseline CHL, HypoCHL participants were more likely to increase their alcohol consumption during the course of the study $(\mathrm{RR}=2.7,95 \% \mathrm{CI}$ 1-7.9, $p=0.045$ ).

As depicted in Table 2, among hazardous drinkers, a significant decline in all drinking measures was observed in the subgroup with normal cholesterol values. In contrast, significant increases over the six months were observed in those with hypocholesterolemia. Further strengthening our findings was the evidence of a doserelationship between baseline cholesterol values and risk of higher alcohol consumption at the last visit (see Table 3).

\section{Final Analyses}

In the final controlled model (i.e. age, race, baseline drinking, CD4) three important results emerged. First, analyses identified among several potential predictors five significant risk factors predicting increases in alcohol use: being a smoker $(\mathrm{OR}=2.995 \%$ CI 1.-6.4, $p=0.008$ ), being a liquor user (OR=1.9 95\% CI 1-4, $p=0.05$ ), having HypoCHL (OR= $1.595 \%$ CI 1-2.8, $p=0.04)$, a close relative with hazardous alcohol use $(\mathrm{OR}=3,95 \%$ CI 1.8 5.6; $p=0.0001$ ), and/or having limited family support. Second, the three-way interaction of cholesterol, depression and smoking was not significant indicating that hypocholesterolemia is an independent risk factor of drinking. Third, the model also reveals that neither depression nor CD4 counts or stage of HIV disease (i.e., symptomatic, asymptomatic or AIDS) significantly alter the probability of increased alcohol use.

To further establish if hypocholesterolemia affected drinking behavior and to determine the variance HypoCHL contributed to predicting alcohol use variables beyond all the other variables in the study, we performed a Bayesian modeling analyses. This modeling data confirms a relationship between baseline cholesterol and subsequent alcohol intake, (i.e., number of days drinking and in number of drinks/day).

Figures 3 and 4 show probabilities associated with the structures when cholesterol is manipulated. This manipulation enabled us to quantify the dynamics of all other variables. For example, the model estimated an $87 \%$ probability of either maintaining or increasing alcohol intake during the study, in the event that baseline cholesterol was low (choles=State1). In this scenario, only $14 \%$ of the participants exhibited reduced alcohol intakes. Noteworthy, when we modeled cholesterol levels to be normal (choles=State), as much as $41 \%$ of the participants were estimated to reduce their alcohol intakes. According to the model, if those with hypocholesterolemia smoked tobacco, only $11 \%$ would be able to quit drinking.

\section{Discussion}

This study provides what appears to be the first evidence in the scientific literature, indicating that hypocholesterolemia may indeed affect drinking behaviors in PLWH. Overall, the considered risk factors explained about $87 \%$ of the attributable risks of maintaining or increasing alcohol intake in PLWH. Over a 6-month follow-up period, an increase in alcohol intake was related to several factors, including smoking, mental health (including depressive symptoms), age, and race. While it is widely acknowledged that alcohol reduces cholesterol levels, both in

Figure 2. Changes in Number of Alcohol Units Consumed per week according to Cholesterol Status.

normal Cholesterol $\quad \mathrm{HypoCHL}$

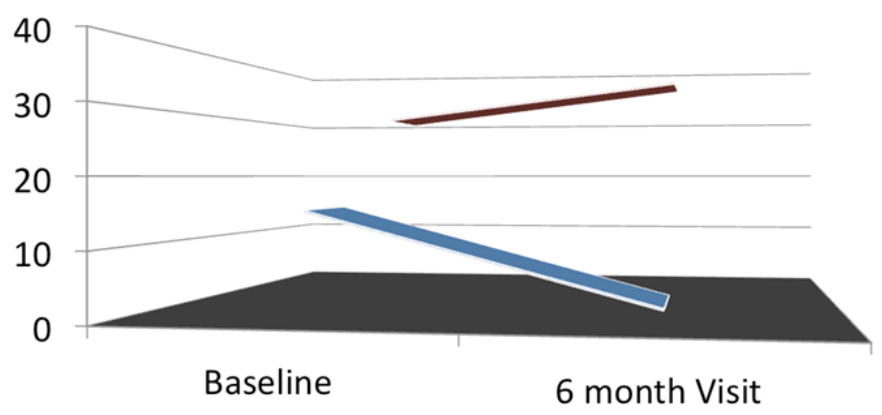

The figure depicts the mean number of Alcohol units consumed over the length of the study, which was significantly higher among the group of subjects with low cholesterol levels. 
Table 2. Mean Changes in Drinking Parameters from Baseline to 6-Month Follow-Up.

\begin{tabular}{|c|c|c|c|c|}
\hline \multirow{2}{*}{} & \multicolumn{2}{|l|}{ Hypocholesterolemia } & \multicolumn{2}{c|}{ Normal Cholesterol } \\
\cline { 2 - 5 } & T2-T1 & P value & T2-T1 & P value \\
\hline Total drinking /week & $+6.4 \pm 2.7$ & 0.02 & $-14 \pm 17$ & 0.001 \\
\hline Number of days per week drinking & $+0.5 \pm 0.3$ & 0.09 & $-1.9 \pm 1$ & 0.000 \\
\hline Drinks per day & $+1.3 \pm 0.6$ & 0.3 & $-1.8 \pm 0.6$ & 0.09 \\
\hline Maximum number of drinks per occasion & $+4.9 \pm 1.7$ & 0.02 & $-8.7 \pm 3.4$ & 0.03 \\
\hline
\end{tabular}

Table 3. Analysis of Cholesterol Dose Response on Maintenance and Increase of Alcohol Consumption.

\begin{tabular}{|c|c|c|c|c|c|}
\hline \multicolumn{6}{|c|}{ Dose Response Analysis Stratum 1} \\
\hline $\begin{array}{c}\text { Exposure Level } \\
\text { Ratio }\end{array}$ & Cases & Controls & Total & $\begin{array}{l}\text { Odds of } \\
\text { Exp. }\end{array}$ & Odds \\
\hline 0 & 2 & 4 & 6 & 0.5 & 1 \\
\hline 1 & 2 & 17 & 19 & 0.12 & 0.24 \\
\hline 2 & 11 & 30 & 41 & 0.37 & 0.73 \\
\hline 3 & 16 & 25 & 41 & 0.64 & 1.28 \\
\hline 4 & 28 & 30 & 58 & 0.93 & 1.87 \\
\hline Total & 59 & 106 & 165 & & \\
\hline $\begin{array}{l}\text { Mantel-Haenszel } \\
\text { Level }\end{array}$ & \multicolumn{5}{|c|}{$\begin{array}{c}\text { Summary Odds Ratios and Crude OR for Each } \\
\text { Exposure }\end{array}$} \\
\hline Exposure MH & \multicolumn{3}{|c|}{ Summary OR } & \multicolumn{2}{|c|}{ Crude OR } \\
\hline Level 0 vs. Level 0 & \multicolumn{3}{|c|}{1} & \multicolumn{2}{|c|}{1} \\
\hline Level 1 vs. Level 0 & \multicolumn{3}{|c|}{0.235} & \multicolumn{2}{|c|}{0.235} \\
\hline Level 2 vs. Level 0 & \multicolumn{3}{|c|}{0.733} & \multicolumn{2}{|c|}{0.733} \\
\hline Level 3 vs. Level 0 & \multicolumn{3}{|c|}{1.28} & \multicolumn{2}{|c|}{1.28} \\
\hline Level 4 vs. Level 0 & \multicolumn{3}{|c|}{1.867} & \multicolumn{2}{|c|}{1.867} \\
\hline
\end{tabular}

Extended Mantel-Haenszel chi square for linear trend $=8.23$ $\mathrm{p}$-value (1 degree of freedom) $=0.004122$

Figure 3. Dynamic Causal Bayesian Networks Normal Cholesterol.
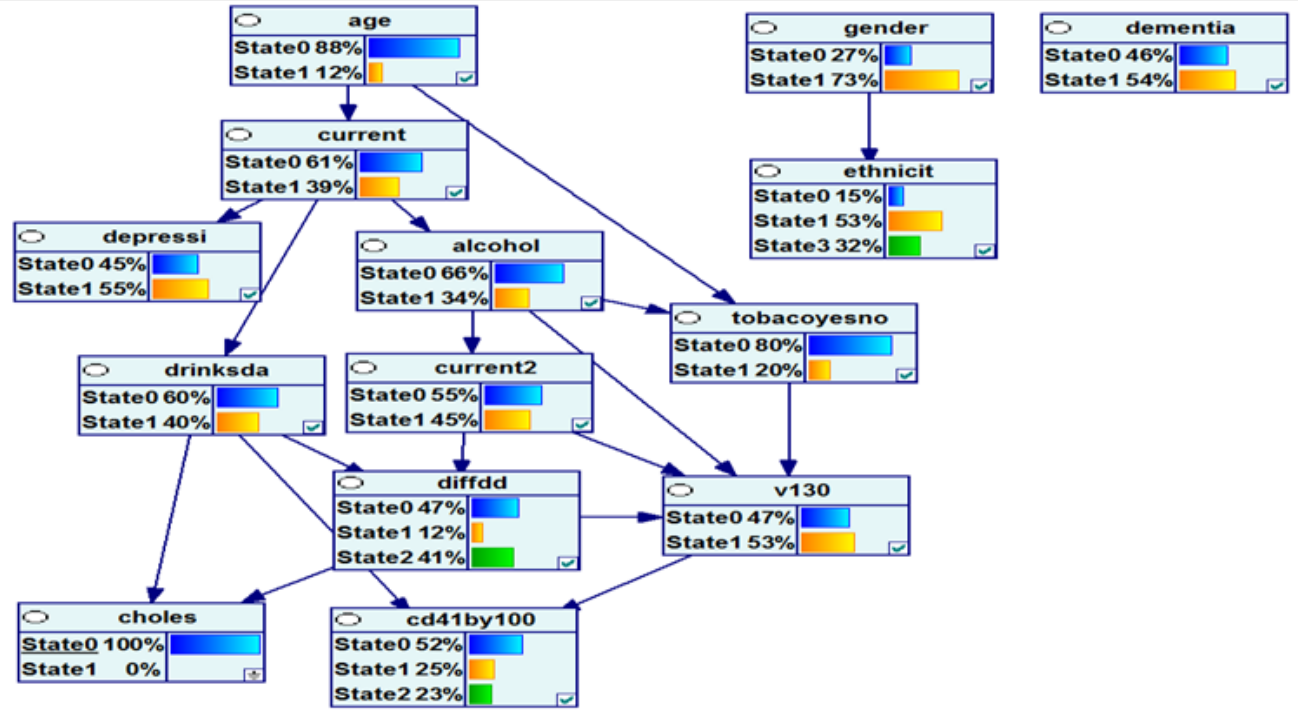

State1 54\%

This modeling data confirms a relationship between baseline cholesterol (choles $0=$ normal vscholes $1=<150)$ and alcohol intake, i.e., number of drinks in a day (drinksda) and difference in number of drinks/day between baseline and final visit (diffdd state $2=$ reduced; state $1=$ maintain and state $0=$ increased). 
Figure 4. Dynamic Causal Bayesian Networks Hypocholesterolemia with Smoking.
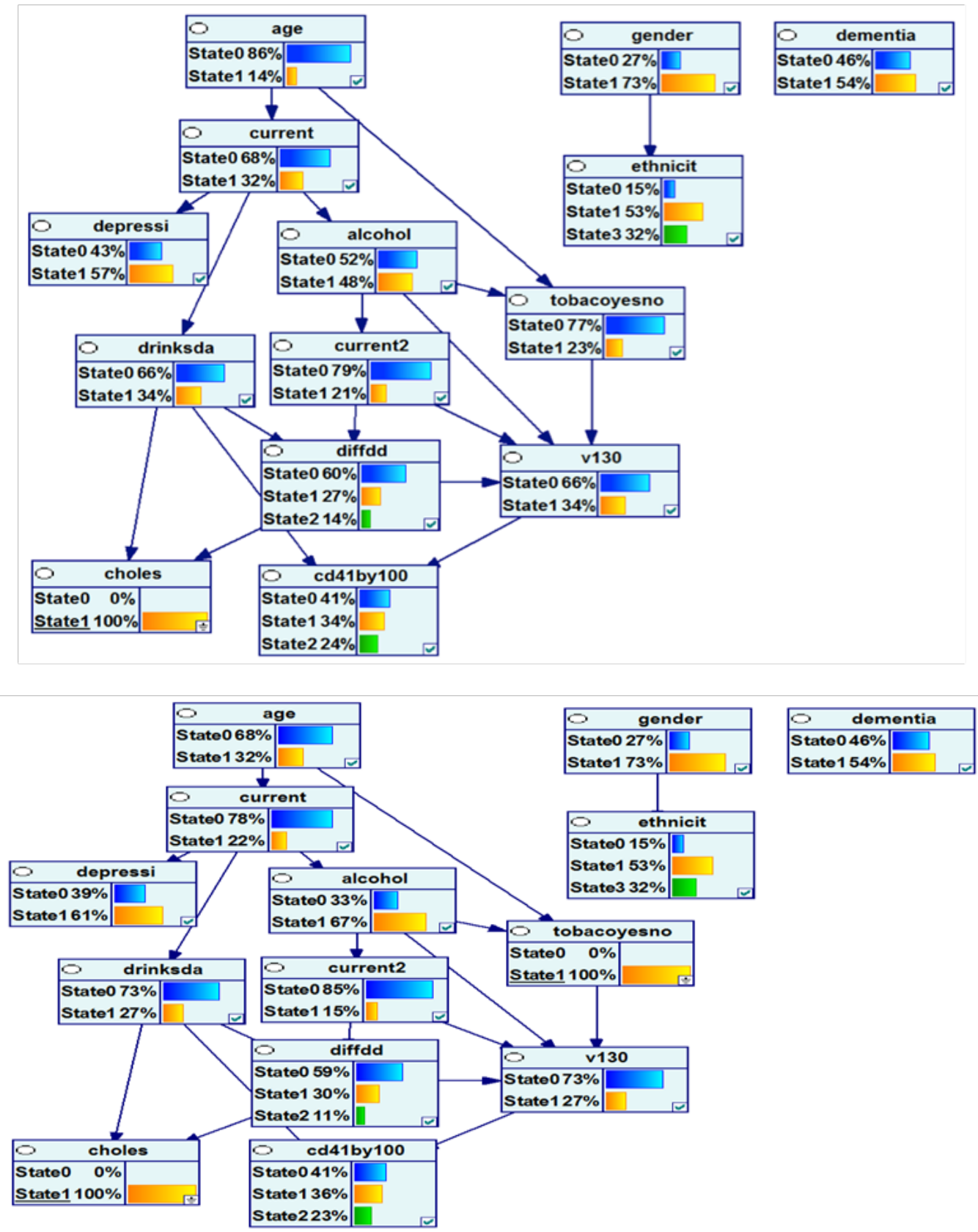

The lower graphic shows the scenario when all subjects smoked and have hypocholesterolemia. Subjects have only $11 \%$ chance of quitting drinking.

plasma as well as in the brain $[40,41]$, the possibility that this relationship is bi-directional has been largely ignored. Although our study design precludes casual inferences, several factors may boost confidence in our findings. First, our model explained a significant proportion of the alcohol trajectories, making it less likely that hypocholesterolemia was merely a moderator of our findings. Second, we performed detailed dose-response and temporal analyses, where baseline values were able to predict results 24 weeks later, thereby strengthening our results. Third, there was a robust and persistent relationship after controlling for confounders supports the notion that they are closely related. Importantly, we controlled for key characteristics common among those who drink alcohol, including sociodemographic (i.e., age, education), psychiatric (i.e., depression), and other behavioral factors (i.e., tobacco). Fourth, while the literature on cholesterol in substance abusers is sparse, Buydens-Branchey and Branchey made a similar observation in cocaine addicts [42]. According to their analyses patients, with HypoCHL at time of admission were more likely to relapse at all-time points $(3,6$, and 12 months after discharge).

These novel associations are likely to be related to cholesterol influences on essential CNS functions, such as signal transduction, synaptogenesis, membrane trafficking, and myelin formation [16, 42-48]. In fact, cholesterol may act by not only causing structural alterations within the biological membranes, but also by changing signal transductions that regulate addictive behaviors such as serotonin, dopamine and the newly discovered sigma-1 receptors $[16,18,24,25,47-52]$. Specifically, studies have repeatedly found that cholesterol may directly alter serotonin synthesis, reuptake 
and postsynaptic serotonin function. These alterations could lead to cognitive impairment, mood disorders and diminished impulse control, all neurobiological features of HAU [11-13, 51]. Cholesterol interactions with dopamine have also been well documented in animal models, and given their critical role in alcohol behaviors, our results are not uncommon or unexpected. [52].

These findings pose several clinical and public health implications. First, our Bayesian model highlights the need to address cholesterol and smoking problems simultaneously to produce an impact on hazardous alcohol use. Interventions can be approached from a dietary standpoint with cholesterol supplements that can be incorporated along with pharmacological or behavioral treatments. The findings also reinforce the notions that even a short educational session, such as the one we provided when informing the participants about the study, can potentially yield considerable results.

However, some limitations in our study should be noted. First, no experimental manipulation was conducted, so our results, while they may be compelling, provide only associations and do not indicate any degrees of causality. While our longitudinal design reinforces the relevance of our results, the short-followup periods limit our capacity to draw stronger, more well-defined conclusions. Findings are strictly limited to the study site, and given the strict exclusion criteria, our study can be criticized from the standpoint of its representativeness. However, these restrictions allowed examination of the potential effects of cholesterol on drinking, though they precluded examination of the potential indirect and interactive effects that might be produced in the presence of such disorders. Finally, we are aware that the study could have been executed in the general population, thus avoiding the confounding effects of HIV disease. However, the striking prevalence of both HAU and hypoCHL among PLWH was the decisive factor, which essentially drove our selection.

The findings of our study should add confidence in the longterm value and applicability of these relationships to the research arena. At best, our analyses open the scientific gateway for studies with longer follow-ups to replicate our findings and to unravel distinct mechanisms underlying the relationship between hypocholesterolemia and alcohol. Considering the dramatic increases in lipid lowering medication prescriptions among PLWH, and the high prevalence of alcohol use disorders [48], these results also emphasize the need for further studies to elaborate the risks and benefits of marked cholesterol reductions in this population group.

\section{References}

[1]. World Health Organization (2011) Global status report on alcohol and health.

[2]. Burnam MA, Bing EG, Morton SC, Sherbourne C, Fleishman JA, et al. (2001) Use of mental health and substance abuse treatment services among adults with HIV in the United States. Arch Gen Psychiatry 58(8): 729-736.

[3]. Galvan FH, Bing EG, Fleishman JA,London AS, Caetano R, et al. (2002) The prevalence of alcohol consumption and heavy drinking among people with HIV in the United States: Results from the HIV Cost and Services Utilization Study. Journal Stud Alcohol. 63(2):179-186.

[4]. Míguez MJ, Shor-Posner G, Morales G, Rodriguez A, and Burbano X (2003) HIV treatment in drug abusers: impact of alcohol use. Addict Biol 8(1): 33-37.

[5]. Bryant KJ (2006) Expanding research on the role of alcohol consumption and related risks in the prevention and treatment of HIV/AIDS. Subst Use Misuse 41(10-12), 1465-507.

[6]. Shuper PA, Neuman, M, Kanteres, F, Baliunas, D, Joharchi, N, et al., (2010) Causal Considerations on Alcohol and HIV/AIDS. A Systematic Review. Alcohol Alcohol. 45(2): 159-166.

[7]. Míguez-Burbano MJ, Lewis JE, Fishman J, Asthana D, and Malow RW (2009) The Influence of Different Types of Alcoholic Beverages on Disrupting HAART. Alcohol Alcoholism. 44(4): 366-371.

[8]. Hahn JAand Samet JH (2010) Alcohol and HIV Disease Progression: Weighing the Evidence. Curr HIV/AIDS Rep 7(4):226-33.

[9]. Brady KT andSinha R (2005) Co-Occurring Mental and Substance Use Disorders: The Neurobiological Effects of Chronic Stress. Am J Psychiatry. 162(8): 1483-1493.

[10]. Cook RL, McGinnis KA, Kraemer KL, Gordon AJ, Conigliaro J, et al. (2006) Intoxication before intercourse and risky sexual behavior in male veterans with and without human immunodeficiency virus infection.Medic Care 44(8): S31-S36.

[11]. Rangaswamy M andPorjesz B (2008) Uncovering genes for cognitive (dys) function and predisposition for alcoholism spectrum disorders: a review of human brain oscillations as effective endophenotypes. Brain Research 1235: $153-71$.

[12]. Patel I (2010) Alcohol and depression. InnovAiT: The RCGP Journal for Associates in Training. 3(3): 155-165.

[13]. Bates ME, Bowden SC,and Barry D. (2002) Neurocognitive impairment associated with alcohol use disorders: implications for treatment. Exp Clin Psychopharmacol. 10(3): 193-212.

[14]. Lesch KP (2005) Alcohol dependence and gene x environment interaction in emotion regulation: Is serotonin the link? Eur J Pharmacol. 526(1-3): 113-24.

[15]. Sari Y, Johnson VR, and Weedman JM (2011) Role of the serotonergic system in alcohol dependence: from animal models to clinics. ProgMolBiolTransl Sci. 98, 401-43.

[16]. Morgan RE, Palinkas LA, Barrett-Connor EL, and Wingard DL (1993) Plasma cholesterol and depressive symptoms in older men. Lancet. 341(8837): $75-79$.

[17]. Engelberg H (1992) Low serum cholesterol and suicide. Lancet. 339(8795): 727-729.

[18]. GolombBA (1998) Cholesterol and violence: is there a connection? Ann Intern Med 128(6): 478-487.

[19]. Kaplan JR, Shively CA, Fontenot MB, Morgan TM, Howell SM, et al. (1994) Demonstration of an association among dietary cholesterol, central serotonergic activity, and social behavior in monkeys. Psychosom Med. 56(6): 479-484.

[20]. Henderson VW, Guthrie JR,and Dennerstein L (2003) Serum lipids and memory in a population based cohort of middle age women. J Neurol Neurosurg Psychiatry 74(11), 1530-1535.

[21]. Reuben DB, Ix JH, Greendale GA, andSeeman TE (1999) The predictive value of combined hypoalbuminemia and hypocholesterolemia in high functioning community-dwelling older persons: MacArthur Studies of Successful Aging. J Am GeriatrSoc. 47(4): 402-406.

[22]. Wada T, Matsubayashi K, Okumiya K, Kimura S, Osaka Y, et al. (1997) Lower serum cholesterol level and later decline in cognitive function in older people living in the community, Japan. J Am GeriatrSoc. 45(11):1411-2.

[23]. Piquet O, Grayson DA, Creasey H, Bennett HP, Brooks WS, et al. (2003) Vascular risk factors, cognition and dementia incidence over 6 years in the Sydney Older Persons Study. Neuroepidemiology. 22(3): 165-171.

[24]. Míguez -Burbano MJ, Lewis J, Burbano X, Rosenberg R, Fishman J, et al. (2010) Variable cognitive benefits in HAART-treated patients: are aging and low cholesterol linked? Neurobehavioral HIV Medicine. 2: 13-21.

[25]. Scanlon SM, Williams DC, and Schloss P (2001) Membrane cholesterol modulates serotonin transporter activity. Biochemistry. 40(35): 10507-13.

[26]. Wrase J, Reimold M, Puls I, Kienast T, and Heinz A (2006) Serotonergic dysfunction: brain imaging and behavioral correlates. Cogn Affect Behav Neurosci. 6(1): 53-61.

[27]. Szabo G, Dolganiuc A, Dai Q, and Pruett SB (2007) TLR4, ethanol, and lipid rafts: a new mechanism of ethanol action with implications for other receptor-mediated effects. J Immunol. 178(3): 1243-1249.

[28]. Lejoyeux M (1996) Use of serotonin (5-hydroxytryptamine) reuptake inhibitors in the treatment of alcoholism Alcohol Alcohol. 31(1): 69-75.

[29]. Babor TF, Higgins-Biddle JC, Saunders JB, and Monteiro MG (2001) AUDIT: The Alcohol Use Disorders Identification Test, 2nd Ed. World Health Organization.

[30]. McLellan AT, Kushner H, Metzger D, Peters R, Smith I, et al., (1992) The Fifth Edition of the Addiction Severity Index. J Subst Abuse Treat, 9(3): 199-213.

[31]. Li TK, Hewitt BG, and Grant BF (2007) The Alcohol Dependence Syndrome, 30 years later: a commentary. Addiction 102(10): 1522-1530. 
[32]. NIAAA and NIH (2010) Rethinking Drinking Booklet: Alcohol and your Health. NIH Publication No. 15-3770.

[33]. Björkhem I and Meaney S (2004) Brain Cholesterol: Long Secret Life Behind a Barrier. Arterioscler Thromb Vasc Biol. 24(5): 806-815.

[34]. National Cholesterol Education Program (2001) ATP 3 Guidelines at Glance: Quick Desk Reference. National Institutes of Health: National Heart, Lung, and Blood Institute. NIH Publication No. 01-3305.

[35]. Beck AT, Ward CH, Mendelson M, Mock J, and Erbaugh J (1961) An inventory for measuring depression. Arch Gen Psychiatry. 4: 561-571.

[36]. Beck AT, Steer RA, Garbin MG (1988) Psychometric properties of the beck depression inventory: Twenty-five years of evaluation. Clin Psychol Rev 8(1): 77-100.

[37]. Cooper GF, Herskovits E (1992) A Bayesian method for the induction of probabilistic networks from data. Mach Learn. 9(4): 309-347.

[38]. Heckerma D, Geiger D, and Chickering D (1995) Learning Bayesian networks: The combination of knowledge and statistical data. Mach Learn. 20(3): 197-243.

[39]. DurazzoTC, Gazdzinski S, Yeh P, and Meyerhoff D (2008) Combined Neuroimaging, Neurocognitive and Psychiatric Factors to Predict Alcohol Consumption Following Treatment for Alcohol Dependence. Alcohol Alcohol. 43(6): 683-691.

[40]. Gustavsson L (1990) Brain lipid changes after ethanol exposure. Ups J Med SciSuppl 48:245-266.

[41]. Agarwal DP (2002) Cardioprotective effects of light-moderate consumption of alcohol: a review of putative mechanisms. Alcohol Alcohol. 37(5): 409-415.

[42]. Buydens-Branchey L and Branchey M (2003) Association Between Low Plasma Levels of Cholesterol and Relapse in Cocaine Addicts. Psychosom Med 65(1): 86-91.

[43]. JawaidA, Poon M, Strutt AM, Rice LK, McDowell EJ, et al. (2011) Does apolipoprotein E genotype modify the clinical expression of ALS? Eur J Neurol. 18(4): 618-624.
[44]. Liu Y, Paajanen T, Westman E, Wahlund LO, Simmons A, et al., (2010) Effect of APOE $\varepsilon 4$ allele on cortical thicknesses and volumes: the AddNeuroMed study. J Alzheimers Dis. 21(3): 947-966.

[45]. Hardwicke RL, Lewis ST, and Grimes RM (2010) Evaluation and Pharmacologic Management of the HIV-Infected Patient with Dyslipidemia. J Assoc Nurses AIDS Care 21(5): 429-438.

[46]. Crowley JJ, Treistman SN, andDopico AM (2003) Cholesterol Antagonizes Ethanol Potentiation of Human Brain BKCa Channels Reconstituted into Phospholipid Bilayers. MolecPharmacol. 64(2): 365-372.

[47]. Yuan C, O'Connell RJ, Wilson A, Pietrzykowski AZ, and Treistman SN (2008) Acute Alcohol Tolerance Is Intrinsic to the BKCa Protein, but is Modulated by the Lipid Environment. J BiolChem. 283(8): 5090-5098.

[48]. Joseph, JA, Villalobos-Molina R, Yamagami K, Roth GS, and Kelly J (1995) Age-specific alterations in muscarinic stimulation of $\mathrm{K}(+)$-evoked dopamine release from striatal slices by cholesterol and S-adenosyl-L-methionine. Brain Research. 673(2):185-193.

[49]. Brown SL, Salive ME, Harris TB,Simonsick EM, Guarlnik JM, et al. (1994) Low cholesterol concentrations and severe depressive symptoms in elderly people. BMJ. 308(6940): 1328-1332.

[50]. Matarazzo S, QuitadamoMC, Mango M, Ciccone S, Novelli G, and Biocca S (2012) Cholesterol-Lowering Drugs Inhibit Lectin-Like Oxidized LowDensity Lipoprotein-1 Receptor Function by Membrane Raft Disruption. Mol. Pharmacol. 82(2): 246-254.

[51]. Grant BF, Stinson FS, Dawson DA, Chou SP, Dufour MC, et al. (2006) Prevalence and Co-Occurrence of Substance Use Disorders and Independent Mood and Anxiety Disorders: Results from the National Epidemiologic Survey on Alcohol and Related Conditions. Arch Gen Psychiatry. 61(8): 807-816.

[52]. Maguire PA and Druse MJ (1989) The influence of cholesterol on synaptic fluidity, dopamine D1 binding and dopamine-stimulated adenylate cyclase. Brain Res Bull. 23(1-2): 69-74. 